\title{
VIOLENCIA LABORAL EXTERNA TIPO AMENAZA CONTRA MÉDICOS EN SERVICIOS HOSPITALARIOS DE LIMA METROPOLITANA, PERU 2014
}

\author{
Ximena Tuya-Figueroa1,a , Edward Mezones-Holguin ${ }^{1,2, \mathrm{~b}}$, Eduardo Monge $^{1,3, \mathrm{c}}$, Ricardo Arones ${ }^{1,4, \mathrm{~d}}$, Milagros Mier ${ }^{1,5, \mathrm{e}}$, \\ Mercedes Saravia ${ }^{1,6, f}$, Jose Torres ${ }^{1,7, g}$, Percy Mayta-Tristán ${ }^{1, h}$
}

\begin{abstract}
RESUMEN
Objetivos. Estimar la frecuencia y los factores asociados a la violencia laboral externa tipo amenaza (VLETA) contra médicos en servicios de salud hospitalarios de Lima Metropolitana, Perú. Materiales y Métodos. Se desarrolló un estudio transversal analítico que incluyó médicos del Ministerio de Salud (MINSA), la Seguridad Social (EsSalud) y el subsector privado. Se midió la frecuencia de VLETA durante toda la práctica profesional, en los últimos doce meses y en el último mes. Se midieron variables relacionadas al médico, al agresor y al servicio de salud. Se estimaron razones de prevalencia (RPs) cruda y ajustada mediante un modelo lineal generalizado familia Poisson con bootstrap no paramétrico. Resultados. Participaron 406 médicos. El 31,5\% fueron víctimas de VLETA al menos una vez durante su práctica profesional; $19,9 \%$ en los últimos doces meses y $7,6 \%$ en el último mes. La probabilidad de ser amenazado en los últimos doce meses aumentó si el médico era varón (RPa:1,7;IC95\%:1,1-2,8), egresado de una universidad peruana fuera de Lima Metropolitana (RPa:1,5; IC95\%:1,1-2,4), laboraba en MINSA (RPa:7,9;IC95\%:2,24-50,73) o en EsSalud (RPa:8,68; IC95\%:2,26-56,17), y atendía en emergencia (RPa:1,9;IC95\%:1,2-3,6) o en sala de operaciones (RPa:1,6 IC95\%:1,1-2,3). La edad, los años de práctica profesional o ser médico residente no se asociaron a VLETA. Conclusiones. En los hospitales estudiados, una alta proporción de médicos ha sido víctima de VLETA. Laborar en servicios públicos aumenta dicha probabilidad. Se sugiere implementar estrategias de prevención primaria, identificación y soporte en los establecimientos hospitalarios.
\end{abstract}

Palabras clave: Violencia laboral; Relaciones médico-paciente; Servicios de salud; Perú (fuente: DeCS BIREME).

\section{EXTERNAL WORKPLACE VIOLENCE AGAINST DOCTORS IN HOSPITAL SERVICES IN LIMA METROPOLITANA, PERU 2014}

\begin{abstract}
Objectives.To calculate the frequency and factors associated with external workplace violence (EWV) against doctors in health inpatient services in the metropolitan area of Lima (Spanish: Lima Metropolitana), Peru. Materials and methods. A cross-sectional analytic study, which included doctors from the Ministry of Health (MINSA), Social Security (EsSalud), and the private subsector, was carried out. The frequency of EWV was measured throughout the entire professional practice during the previous 12 months and during the last month. Variables related to the doctor, assailant, and health service were measured. Raw and adjusted prevalence ratios (PR) were calculated by means of a Poisson-family generalized linear model with non-parametric bootstrapping. Results. A total of 406 doctors participated; $31.5 \%$ were victims of EWV at least once during their professional practice, with $19.9 \%$ over the past 12 months and $7.6 \%$ during the last month. The chances of being threatened in the last 12 months increased if the doctor was male (adjusted PR [aPR]: 1.7; 95\% confidence interval [Cl] $=1.1$ 2.8), had graduated from a Peruvian university outside of the metropolitan area of Lima (aPR: $1.5 ; 95 \% \mathrm{Cl}=1.1-2.4$ ), worked at MINSA (aPR: $7.9 ; 95 \% \mathrm{Cl}=2.24-50.73$ ) or EsSalud (RR: 8.68; 95\% Cl = 2.26-56.17), and worked in the emergency (aPR: $1.9 ; 95 \% \mathrm{Cl}=1.2-3.6$ ) or operating room (aPR: $1.6 ; 95 \% \mathrm{Cl}=1.1-2.3$ ). Age, years of professional practice, or being a medical resident were not associated with EWV. Conclusion. In the hospitals studied, a large number of doctors have been victims of EWV. Working in public services increases the possibility of violence. Implementation of support, identification, and primary prevention strategies in hospitals is recommended.
\end{abstract}

Keywords: Workplace Violence; Physician-patient relations; Health services; Peru (source: MeSH NLM).

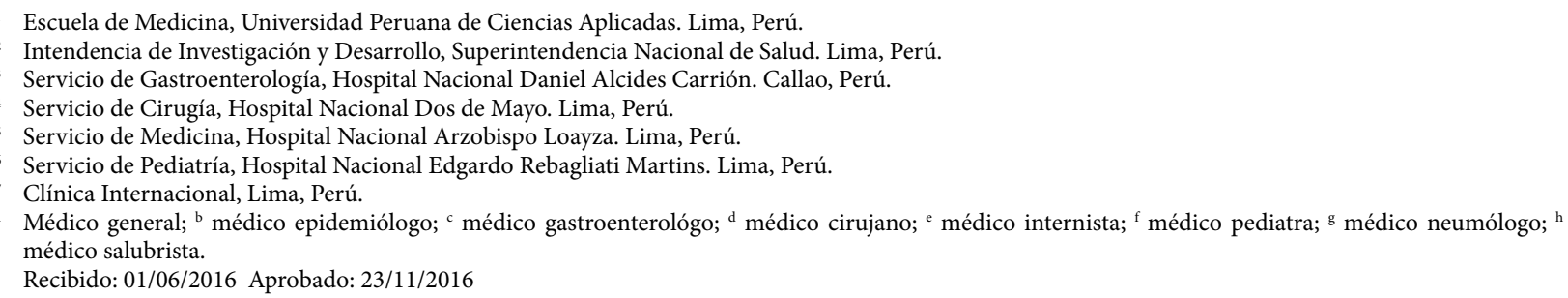

Citar como: Tuya-Figueroa X, Mezones-Holquin E, Monge E, Arones R, Mier M, Saravia M, Torres J, Mayta-Tristán P. Violencia laboral externa tipo amenaza contra médicos en servicios hospitalarios de Lima Metropolitana, Perú 2014. Rev Peru Med Exp Salud Publica. 2016;33(4):670-9. doi: 10.17843/rpmesp.2016.334.2550 


\section{INTRODUCCIÓN}

La violencia laboral (VL) es un problema que ocurre tanto en países industrializados como en vías de desarrollo (1). La VL está definida como toda acción o incidente no razonable donde un trabajador es agredido, amenazado, humillado o lesionado en el ejercicio de su actividad profesional o como consecuencia directa de la misma ${ }^{(2)}$. La VL externa surge de la interacción entre el trabajador y una persona con la que no guarda vínculo laboral, pero se encuentra en el lugar de trabajo ${ }^{(3)}$. Si bien la VL física es identificada con mayor facilidad, la VL psicológica, aunque menos reportada, suele ser la más frecuente ${ }^{(4)} \mathrm{y}$, dentro de esta, es la violencia laboral externa tipo amenaza (VLETA) la que constituye una fuente importante de estrés para la víctima ${ }^{(5)}$. Consecuentemente, la VLETA es un tópico de alto interés que requiere ser estudiado, más aun cuando puede comportarse de manera distinta de acuerdo al ámbito laboral que se investigue.

La VL puede presentarse en diversos grupos ocupacionales, y el sector Salud es uno de los más afectados ${ }^{(6,7)}$. A nivel mundial, la cuarta parte de los casos de VL sucede en el área de salud, y uno de cada dos profesionales de salud es víctima de violencia ${ }^{(6)}$. En Estados Unidos de América, un profesional de la salud tiene dieciséis veces la probabilidad de ser víctima de VL, en comparación con profesionales de otras áreas ${ }^{(6)}$. En América Latina se ha descrito una frecuencia de más de $50 \%$ en base a una encuesta en Internet ${ }^{(8)}$. En Perú se reportó que dos tercios de los médicos de un hospital de Huaraz fueron víctimas de algún tipo de violencia ${ }^{(9)}$, el $84 \%$ de médicos de tres hospitales de Arequipa sufrieron violencia alguna vez en su vida ${ }^{(10)}$ y que el $72 \%$ de médicos de un hospital de Tacna sufrieron algún tipo de agresión (11). Por tanto, la VL externa sobre los médicos es un problema emergente, y requiere investigaciones que evalúen los factores asociados a este fenómeno.

El estudio de los factores asociados a la VL resulta complejo, puesto que entrelaza diversos aspectos del profesional de salud, del paciente y de los sistemas de salud ${ }^{(7,10,12-14)}$ tales como la edad, la especialidad médica y el sexo del médico ${ }^{(7,12)}$, así como el estado de salud, el sexo del paciente o el efecto de sustancias como el alcohol ${ }^{(7,12-14)}$. No obstante, los aspectos concernientes al sistema de salud han sido poco investigados ${ }^{(7)}$, más aun en escenarios con sistemas de salud fragmentados como el peruano ${ }^{(15)}$

Los efectos de la VL sobre los médicos se dan a diversos niveles ${ }^{(7)}$. Se reflejarían en la prestación del servicio de salud afectando la calidad en los cuidados y la relación con el paciente; asimismo, perjudicarian la satisfacción del profesional e, incluso, conllevar al abandono de la profesión ${ }^{(2,7)}$. Paralelamente, se generarían impactos económicos, pues se conoce que el estrés y la violencia producen casi el $30 \%$ de los costos totales de las enfermedades y accidentes, y sus consecuencias comprometen entre 0,5 y $3,5 \%$ del producto bruto interno anual (1).

Por lo mencionado, evaluar el comportamiento de la VLETA en médicos se convierte en una necesidad, y más cuando el estudio de la gestión de recursos humanos en salud es una de las prioridades de investigación en nuestro país ${ }^{(16)}$. El objetivo de este estudio fue estimar la frecuencia y los factores asociados de la violencia laboral externa tipo amenaza sobre los médicos de los servicios de salud hospitalarios de Lima Metropolitana. Los resultados obtenidos pueden contribuir al planteamiento de políticas en el sector salud, al desarrollo de intervenciones para prevenir agresiones y a la formulación de nuevas investigaciones.

\section{MATERIAL Y MÉTODOS}

\section{DISEÑO}

Se desarrolló un estudio transversal analítico durante los meses de marzo a julio de 2014 en establecimientos hospitalarios de Lima Metropolitana, Perú.

\section{POBLACIÓN Y MUESTRA}

Se incluyeron a médicos que laboraban en los hospitales del Ministerio de Salud (MINSA): Hospital Nacional Daniel Alcides Carrión (HNDAC), Hospital Nacional Arzobispo Loayza (HNAL) y Hospital Nacional Dos de Mayo (HNDM); de la seguridad social (EsSalud): Hospital Nacional Edgardo Rebagliati Martins (HNERM) y uno del subsector privado (Clínica Internacional). Se excluyó a los médicos que no habían establecido relación con el paciente debido a su especialidad o por trabajar en áreas administrativas, y a quiénes solo estaban haciendo rotaciones cortas en los establecimientos. Asimismo, los cuestionarios incompletos y no retornados fueron excluidos del análisis.

La muestra fue calculada con el software nQuery Advisor ® (Statistical Solutions, Cork, Irlanda) para efectuar un modelo de regresión para desenlaces dicotómicos, con un $95 \%$ de nivel de confianza para una prueba a dos colas y una potencia estadística de $80 \%$, un coeficiente $\beta$ de 0,699 y una frecuencia de desenlace del $50 \%$ para poder ajustar 
un máximo de ocho covariables, con lo que se estimó una muestra mínima de 381 médicos. Además, al considerar los resultados del estudio piloto ${ }^{(9)}$, se calculó una proporción de rechazo de $13,16 \%$, un $2,63 \%$ de cuestionarios no retornados y un $5,26 \%$ de cuestionarios respondidos de modo incompleto; con ello se fijó que el número mínimo de médicos que se requería invitar a participar era 461. La selección de los establecimientos de salud y de los médicos fue no probabilística.

\section{VARIABLES Y MEDICIONES}

Para la medición de violencia se tomaron las preguntas estandarizadas sobre violencia física, psicológica y acoso sexual de la Organización Internacional del Trabajo ${ }^{(17)}$, específicamente, dentro de la subsección de violencia psicológica, la amenaza fue analizada en el presente artículo. La amenaza se definió como la promesa del uso de fuerza o influencias para generarle consecuencias negativas al médico, pudiendo ser estas físicas, psicológicas o sexuales, donde el agresor podría ser el paciente, el familiar o el cuidador ${ }^{(5)}$.

El cuestionario fue autoadministrado, previamente evaluado en un estudio piloto (9) y estuvo compuesto por cuatro secciones. La primera sección exploró la frecuencia de VLETA, en la cual se incluyeron variables como ser testigo del incidente (sí/no) y haber sido víctima alguna vez durante la práctica profesional (sí/ no), en los últimos doce meses (sí/no) y en el último mes (sí/no).

La segunda sección evaluó variables relacionadas al médico: edad (en años), sexo (varón/mujer), estado civil (con pareja/sin pareja), descendencia (con hijos/ sin hijos), afiliación religiosa (sí/no -ateo/agnóstico-), migración registrada según la ciudad en la que nació, finalizó la educación secundaria y ciudad en la que terminó la universidad (Lima Metropolitana/otra provincia del Perú/ el extranjero); la condición laboral (asistente/residente), tener especialidad (sí/no), el tipo de especialidad (quirúrgica/clínica/ayuda al diagnóstico y laboratorio), los años de práctica profesional y el ejercicio de la docencia universitaria (sí/no).

En la tercera sección se incluyeron las variables relacionadas al agresor: sexo del agresor (mujer/varón), tipo de agresor (paciente/familiar/cuidador), estado de salud (estable/de cuidado/ grave/ muy grave/fallecido) y efecto de drogas u otras sustancias (sí/no).

En la cuarta sección se evaluaron aspectos del servicio de salud: el subsector donde labora el médico
(Privado/MINSA/EsSalud), las áreas en que atiende (consultas/emergencia/hospitalización/gerencia/sala de operaciones $/ \mathrm{UCl}$ /imágenes /laboratorio y patología), el lugar del incidente (dentro del consultorio/ dentro del cuarto del paciente/ en sala de espera/corredores del hospital/ emergencia/ oficina) y el turno en el que se produjo la agresión (mañana/tarde/noche).

Los datos fueron recogidos por estudiantes de Medicina previamente entrenados para tal fin, quienes tuvieron constante comunicación con una de las autoras del estudio, quien también supervisó el trabajo de campo. La administración de las encuestas no interfirió con el trabajo del médico. Se informó sobre los objetivos de la investigación y se enfatizó que los incidentes reportados debían ser solo aquellos relacionados al lugar donde se entregó el cuestionario.

\section{ANÁLISIS ESTADÍSTICO}

Se generó una base de datos mediante doble digitación en Excel 2010 ® (Microsoft Corporation, CA, USA), luego del control de calidad se exportó a STATA versión $12.0 \AA$ (STATA Corp, Station College, TXT, USA). Las variables categóricas se presentaron en frecuencias absolutas y porcentajes, para las variables numéricas se usó mediana y desviación intercuartílica debido al no cumplimiento de la normalidad evaluada con la prueba de Shapiro Wilk.

La variable de respuesta fue definida como la VLETA recibida en los últimos doce meses. Se evaluó su asociación con los factores medidos usando la prueba de chi cuadrado o la prueba exacta de Fisher, según correspondía. Posteriormente, se evaluó el mejor modelo de regresión múltiple que podría ajustar los datos analizados; sin embargo, no se encontró el cumplimiento de los supuestos correspondientes, fundamentalmente por no cumplir el tener una distribución conocida poblacional. Asimismo, existían observaciones reducidas en ciertas categorías de variables.

Ante ello, se optó por efectuar la estimación de las medidas de asociación y su incertidumbre mediante bootstrap no paramétrico, una técnica de remuestreo (resampling) que no requiere el desarrollo y cumplimiento de los supuestos acerca de la estructura probabilística de las observaciones. Esta estrategia permite tener estimaciones más precisas y menos sesgadas (sesgo estadístico) de los parámetros de interés. Para estimar los intervalos de confianza se efectuaron 1000 réplicas con el método de sesgo corregido y acelerado (biascorrected and accelerated bootstrap) ${ }^{(18)}$ para un modelo 
lineal generalizado de familia Poisson link log con errores estándar robustos. Se generaron modelos crudos entre los factores estudiados y la variable de respuesta. Finalmente, se construyó un modelo de regresión de múltiples variables ingresando aquellas significativas en el análisis bivariado simple y en el bootstrap crudo.

\section{ASPECTOS ÉTICOS}

El protocolo fue aprobado por el Comité de Ética e Investigación de la Universidad Peruana de Ciencias Aplicadas, del HNAZ y el HNDM, así como de las oficinas de apoyo a la docencia e investigación del HNDAC y HNERM y, la autorización de la Clínica Internacional. Además, se informó a los participantes sobre el alcance de la investigación. La participación fue anónima y voluntaria, previo consentimiento informado escrito.

\section{RESULTADOS}

\section{SELECCIÓN DE LOS PARTICIPANTES}

Se invitó a participar a 558 médicos, 84 del subsector privado, 192 de EsSalud y 282 del MINSA, de los cuales el $18,8 \%$ rechazaron participar en la investigación. Finalmente, se incluyeron a 406 participantes (Figura 1).

\section{ANÁLISIS DESCRIPTIVO}

La mediana de edad y de tiempo de práctica profesional fue 38 y 12 años, respectivamente. La mayoría (64,3\%) fueron varones, $53,7 \%$ nacieron en Lima Metropolitana (LM) y 70,4\% culminaron sus estudios de pregrado en LM. El $54,4 \%$ de los participantes reportaron tener pareja y el $59,6 \%$ tenía hijos. El 9,6\% reportó ser ateo o agnóstico. El $69,2 \%$ eran médicos asistentes, el $36,7 \%$ tenían una especialidad quirúrgica y el $47 \%$ ejercían la docencia universitaria (Tabla 1).

La mayoría de médicos $(59,6 \%)$ reportó haber sido testigo de VLETA a sus colegas, $31,5 \%$ sufrieron VLETA alguna vez durante su práctica profesional, $19,9 \%$ en los últimos doces meses y $7,6 \%$ durante el último mes. El turno en el que más agresiones se reportaron fue la mañana, y el lugar donde ocurrieron con más frecuencia fue la sala de emergencias. Los familiares de los pacientes fueron quienes amenazaron a los médicos con más frecuencia $(75,3 \%)$, más de la mitad de agresores fueron varones y cerca de un décimo de los agresores estuvo bajo el efecto del alcohol u otras sustancias. El $65,4 \%$ de hechos estaba vinculado a pacientes estables (Tabla 2).

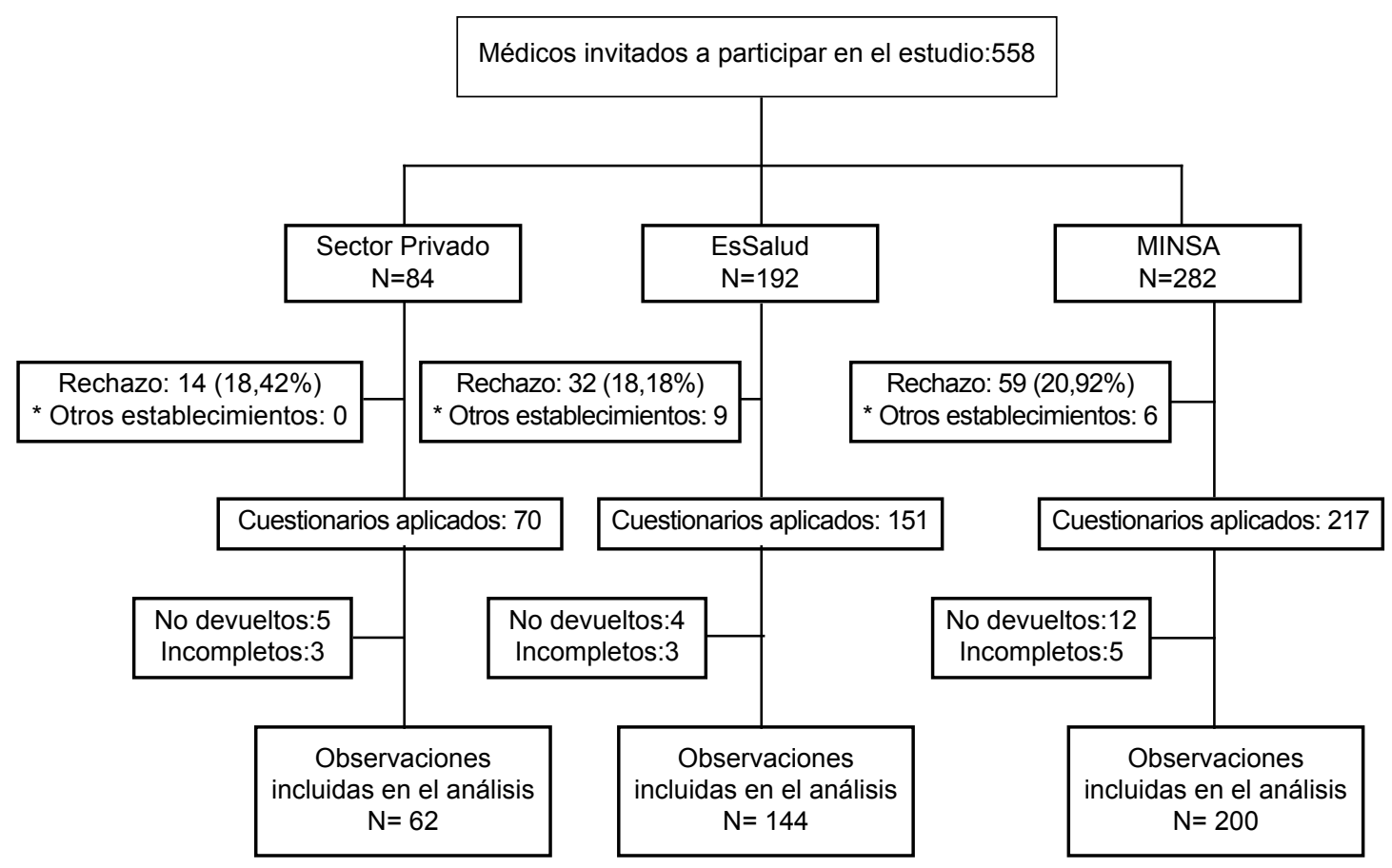

Figura 1. Flujo general de la selección de participantes incluidos en el análisis. *Médicos de otros hospitales que se encontraban haciendo rotaciones.

MINSA: Ministerio de Salud del Perú, EsSalud: Seguro Social de Salud. 
Tabla 1. Características generales de los médicos de hospitales de Lima Metropolitana incluidos en el análisis $(n=406)$

\begin{tabular}{|c|c|c|}
\hline Características & $\mathbf{n}$ & $(\%)^{*}$ \\
\hline Edad (años) $)^{\star *}$ & 38 & $(10)$ \\
\hline$<30$ años & 84 & $(20,7)$ \\
\hline 30 a 39 años & 130 & $(32,0)$ \\
\hline 40 a 49 años & 78 & $(19,2)$ \\
\hline 50 a 59 años & 72 & $(17,7)$ \\
\hline$\geq 60$ años & 42 & $(10,3)$ \\
\hline \multicolumn{3}{|l|}{ Sexo } \\
\hline Varón & 261 & $(64,3)$ \\
\hline Mujer & 145 & $(35,7)$ \\
\hline \multicolumn{3}{|l|}{ Ciudad de nacimiento } \\
\hline Lima Metropolitana & 218 & $(53,7)$ \\
\hline Otras provincias & 185 & $(45,6)$ \\
\hline En el extranjero & 3 & $(0,7)$ \\
\hline \multicolumn{3}{|l|}{ Ciudad donde terminó la carrera } \\
\hline Lima Metropolitana & 286 & $(70,4)$ \\
\hline Otras provincias & 114 & $(28,1)$ \\
\hline En el extranjero & 6 & $(1,5)$ \\
\hline \multicolumn{3}{|l|}{ Estado civil } \\
\hline Con pareja & 221 & $(54,4)$ \\
\hline Sin pareja & 185 & $(45,6)$ \\
\hline \multicolumn{3}{|l|}{ Hijos } \\
\hline Sí & 242 & $(59,6)$ \\
\hline No & 164 & $(40,4)$ \\
\hline \multicolumn{3}{|l|}{ Religión } \\
\hline Ateo/agnóstico & 39 & $(9,6)$ \\
\hline No agnóstico & 367 & $(90,4)$ \\
\hline \multicolumn{3}{|l|}{ Condición } \\
\hline Médico asistente & 281 & $(69,2)$ \\
\hline Médico residente & 125 & $(30,8)$ \\
\hline \multicolumn{3}{|l|}{ Especialidad $^{* \star *}$} \\
\hline Especialidad quirúrgica & 149 & $(36,7)$ \\
\hline Especialidad no quirúrgica & 257 & $(63,3)$ \\
\hline Años de práctica profesional $\left.\right|^{* *}$ & 12 & (9) \\
\hline Menos de 10 años & 191 & $(47,0)$ \\
\hline 10 a 19 años & 85 & $(20,9)$ \\
\hline 20 a 29 años & 77 & $(29,0)$ \\
\hline Más de 30 años & 53 & $(13,1)$ \\
\hline \multicolumn{3}{|l|}{ Docencia } \\
\hline Sí & 191 & $(47,0)$ \\
\hline No & 215 & $(53,0)$ \\
\hline $\begin{array}{ll}* & \mathrm{n}(\%)=\text { frecuencia absoluta (po } \\
* * & \text { mediana (desviación cuartílica) } \\
* * * & \text { Los médicos residentes fuero } \\
& \text { especialidad de formación. }\end{array}$ & rizados & uerdo \\
\hline
\end{tabular}

\section{ANÁLISIS BIVARIADO}

El trabajar en establecimientos de MINSA $(55,6 \%)$ o EsSalud $(43,2 \%)$, atender en emergencias $(80,3 \%)$ o sala de operaciones $(44,4 \%)$, tener una especialidad quirúrgica $(55,6 \%)$, ser residente $(44,4 \%)$, haber concluido el pregrado en provincias (42\%) y ser varón $(76,5 \%)$ mostraron una mayor frecuencia significativa de VLETA durante los últimos doce meses $(p<0,05)$ (Tabla 3). En la Figura 2 se muestran las frecuencias relativas de víctimas de VLETA para cada subsector evaluado.

\section{ANÁLISIS DE MÚLTIPLES VARIABLES}

Se encontró que los médicos varones (RPa: 1,66; IC95\%:1,05-2,96 y aquellos que concluyeron el pregrado en una universidad peruana fuera de Lima (RPa: 1,54; IC95\%: 1,15-2,54) tenían una probabilidad mayor de haber sido víctimas de VLETA en los últimos doce meses. También aquellos que laboraban en EsSalud (RPa: 8,68; IC95\%:2,26-56-17) y el MINSA (RPa: 7,98 IC95\%: 2,24-50,73) tuvieron una mayor probabilidad de VLETA en los últimos doce meses comparados con los que trabajaban en la clínica privada. De manera similar, el trabajar en emergencia (RPa: 1,93; IC95\%: 1,24-3,55) y en sala de operaciones (RPa: 1,66; IC95\%: 1,07-2,34) (Tabla 4).

Resaltamos que debido a que existió una alta colinealidad entre el haber concluido el colegio y la universidad en una provincia fuera de Lima, solo se consideró esta última variable. Por el mismo motivo se decidió solo incluir en el análisis el área de trabajo y no especialidad. El ser residente no fue incluido en el

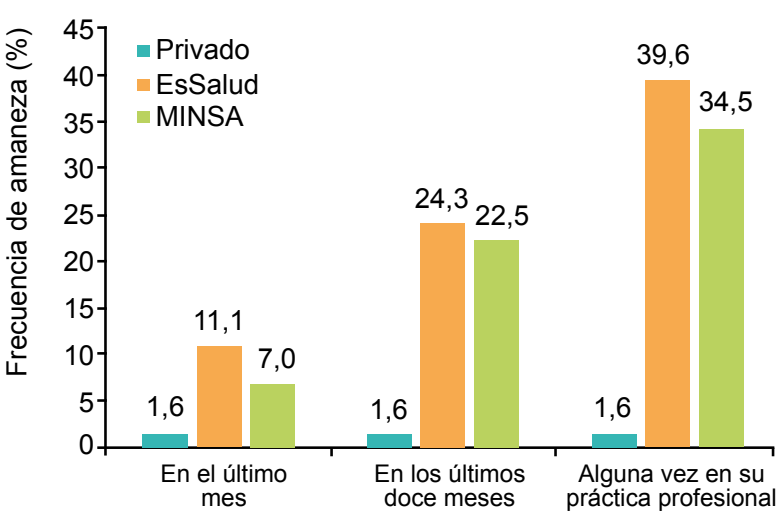

MINSA: Ministerio de Salud del Perú, EsSalud: Seguro Social de Salud.

Figura 2. Frecuencia de violencia psicológica tipo amenaza según tipo de hospital en los médicos de Lima Metropolitana, 2014. 
Tabla 2. Frecuencia y características de la violencia psicológica tipo amenaza contra médicos de hospitales de Lima Metropolitana, 2014

\begin{tabular}{|c|c|c|}
\hline Característica & $\mathbf{n}$ & $(\%)$ \\
\hline \multicolumn{3}{|c|}{$\begin{array}{l}\text { Frecuencia de eventos de violencia tipo amenaza en los } \\
\text { sujetos estudiados }(n=406)\end{array}$} \\
\hline \multicolumn{3}{|c|}{ Como víctima } \\
\hline Alguna en su práctica profesional & 128 & $(31,5)$ \\
\hline Durante los últimos doce meses & 81 & $(19,9)$ \\
\hline En el último mes & 31 & $(7,6)$ \\
\hline \multicolumn{3}{|l|}{ Como testigo } \\
\hline $\begin{array}{l}\text { Alguna vez en su práctica } \\
\text { profesional }\end{array}$ & 242 & $(59,6)$ \\
\hline \multicolumn{3}{|c|}{$\begin{array}{l}\text { Características del último hecho violento en los } 12 \text { meses } \\
\text { previos }(n=81)\end{array}$} \\
\hline \multicolumn{3}{|c|}{ Agresor } \\
\hline Paciente & 17 & $(20,9)$ \\
\hline Familiar & 61 & $(75,3)$ \\
\hline Paciente y familiar & 3 & $(3,7)$ \\
\hline \multicolumn{3}{|l|}{ Sexo de agresor y cantidad } \\
\hline Una mujer & 27 & $(33,3)$ \\
\hline Un varón & 46 & $(56,8)$ \\
\hline Más de una mujer & 2 & $(2,5)$ \\
\hline Más de un varón & 1 & $(1,2)$ \\
\hline Mujer y varón & 5 & $(6,2)$ \\
\hline \multicolumn{3}{|c|}{ Uso de drogas o alcohol por parte del agresor ${ }^{\star *}$} \\
\hline Sí & 8 & $(9,9)$ \\
\hline \multicolumn{3}{|c|}{ Estado del paciente relacionado al agresor } \\
\hline Estable & 53 & $(65,4)$ \\
\hline De cuidado & 14 & $(17,3)$ \\
\hline Grave & 10 & $(12,4)$ \\
\hline Muy grave & 2 & $(2,5)$ \\
\hline Fallecido & 2 & $(2,5)$ \\
\hline \multicolumn{3}{|l|}{ Turno } \\
\hline Mañana & 39 & $(48,2)$ \\
\hline Tarde & 14 & $(17,3)$ \\
\hline Noche & 28 & $(34,6)$ \\
\hline \multicolumn{3}{|l|}{ Servicio } \\
\hline Consulta ambulatoria & 14 & $(17,3)$ \\
\hline Emergencia & 46 & $(56,8)$ \\
\hline Hospitalización & 20 & $(24,7)$ \\
\hline Unidad de cuidados intensivos & 1 & $(1,2)$ \\
\hline
\end{tabular}

*Las frecuencias relativas se han calculado en base a los agredidos en el último año, si se tuvo más de una agresión se consideró la última. ** Percepción por parte del médico, corresponde cuando menos a uno de los agresores en los casos en que la agresión fue efectuada por más de una persona

modelo final, debido a que en el análisis con bootstrap crudo no salió asociado y generaba inestabilidad al incluirse en el modelo final.

\section{DISCUSIÓN}

Aunque los servicios de salud, las situaciones sociales y económicas son particulares en cada país, en este estudio se observó que la VLETA afecta a los médicos en los servicios hospitalarios de LM y se han identificado factores asociados a ello. Se halló que los médicos que trabajaban en los hospitales públicos estudiados (MINSA y EsSalud) tenían mayor probabilidad de haber sufrido VLETA en los últimos doce meses, en comparación los médicos del subsector privado. Este resultado es consistente un reporte previo que señala que la VLE contra médicos es menos frecuente en los establecimientos privados ${ }^{(13)}$. Una de las posibles explicaciones de este hallazgo podría estar relacionado a la satisfacción del paciente; por ejemplo, se ha reportado que más del $50 \%$ de los pacientes de emergencia en los establecimientos de salud de EsSalud no están satisfechos con la atención brindada ${ }^{(19)}$. El paciente insatisfecho podría ver en la violencia contra el médico una forma de protesta y demanda de mejor atención ${ }^{(14)}$. Adicionalmente, el nivel educativo del paciente también podría tener un efecto en la forma de dirigirse, presentar un reclamo o exteriorizar la insatisfacción.

Se observó que, dentro de los establecimientos de salud, el área de emergencia es el lugar donde la VLETA ocurrió con mayor frecuencia, lo cual coincide con reportes anteriores ${ }^{(20-22)}$. La agudeza y la gravedad de los pacientes en esta área se han descrito como una de las posibles causas ${ }^{(23)}$. Asimismo, los médicos que laboran en sala de operaciones y en el área quirúrgica también tuvieron mayor probabilidad de ser amenazados. Esto concuerda con investigaciones que han identificado a los médicos con especialidades quirúrgicas como las víctimas más frecuentes ${ }^{(7,12)}$ y señalan como posibles causas las implicaciones propias de los procedimientos quirúrgicos y las largas esperas para programación ${ }^{(7)}$.

Llama la atención que, a diferencia de los antecedentes que reportan que la mayoría de agresiones ocurren en el turno nocturno ${ }^{(20)}$, en este estudio la mayoría de agresiones ocurrieron en el turno de la mañana. Los estudios previos realizados en Perú sobre el problema han sido descriptivos ${ }^{(9-11)}$ o no se cuenta con información para realizar comparaciones o explicar la causa de esta diferencia ${ }^{(24)}$. No obstante, podría existir un sesgo en la selección que subestime los eventos nocturnos de VLETA debido a que el estudio se ejecutó en la mañana y la tarde.

Se identificó que el agresor más frecuente es el familiar del paciente atendido, similar al resultado de otras series ${ }^{(8,25,26)}$. Esto ocurriría debido a que el paciente en su estado de enfermedad no suele agredir a su médico tratante y a que durante el tratamiento suele existir una relación médico-paciente favorable. Sin embargo, no siempre se establece una relación de colaboración médico-paciente-familia y en este caso el familiar poco involucrado e informado en el proceso de atención tendería a atacar al médico. Por tanto, el médico debería 
Tabla 3. Asociación entre características del médico y violencia psicológica tipo amenaza durante los últimos doce meses en médicos de hospitales de Lima Metropolitana, 2014

\begin{tabular}{|c|c|c|c|c|c|}
\hline \multirow[t]{2}{*}{ Características } & \multicolumn{2}{|c|}{$\begin{array}{c}\text { Amenazado } \\
(n=81)\end{array}$} & \multicolumn{2}{|c|}{$\begin{array}{c}\text { No amenazado } \\
(n=325)\end{array}$} & \multirow[t]{2}{*}{ Significancia } \\
\hline & \multicolumn{2}{|c|}{ n (\%) } & \multicolumn{2}{|c|}{ n (\%) } & \\
\hline & & & & & \\
\hline$<30$ años & 25 & $(30,9)$ & 59 & $(18,2)$ & \\
\hline 30 a 39 años & 23 & $(28,4)$ & 107 & $(32,9)$ & \\
\hline 40 a 49 años & 13 & $(16,0)$ & 65 & $(20)$ & \\
\hline 50 a 59 años & 13 & $(16,0)$ & 59 & $(18,2)$ & \\
\hline$\geq 60$ años & 7 & $(8,6)$ & 35 & $(10,8)$ & \\
\hline Varón & 62 & $(76,5)$ & 199 & $(61,2)$ & 0,010 \\
\hline Con pareja & 44 & $(54,3)$ & 177 & $(54,5)$ & 0,982 \\
\hline Tiene hijos & 45 & $(55,6)$ & 197 & $(60,6)$ & 0,406 \\
\hline Ateo/Agnóstico & 10 & $(12,4)$ & 29 & $(8,9)$ & 0,350 \\
\hline \multicolumn{6}{|l|}{ Migración } \\
\hline Nació en provincias & 42 & $(51,9)$ & 143 & $(44,0)$ & 0,204 \\
\hline Concluyó el pregrado en provincias & 34 & $(42,0)$ & 80 & $(24,6)$ & 0,002 \\
\hline \multicolumn{6}{|l|}{ Años de práctica médica } \\
\hline$<10$ años & 44 & $(54,3)$ & 147 & $(45,2)$ & 0,191 \\
\hline 10 a 19 años & 16 & $(19,8)$ & 69 & $(21,2)$ & \\
\hline 20 a 29 años & 11 & $(13,6)$ & 66 & $(20,3)$ & \\
\hline$\geq 30$ años & 10 & $(12,3)$ & 43 & $(13,2)$ & \\
\hline Especialidad quirúrgica & 45 & $(55,6)$ & 104 & $(32,0)$ & $<0,001$ \\
\hline Médico residente & 36 & $(44,4)$ & 89 & $(27,4)$ & 0,003 \\
\hline Realiza actividad docente & 36 & $(44,4)$ & 155 & $(47,7)$ & 0,600 \\
\hline Lugar de trabajo* & & & & & $<0,001$ \\
\hline Hospital MINSA & 45 & $(55,6)$ & 155 & $(47,7)$ & \\
\hline Hospital EsSalud & 35 & $(43,2)$ & 109 & $(33,5)$ & \\
\hline Clínica privada & 1 & $(1,2)$ & 61 & $(18,8)$ & \\
\hline \multicolumn{6}{|l|}{ Área de trabajo } \\
\hline Consulta & 57 & $(70,4)$ & 226 & $(69,5)$ & 0,884 \\
\hline Emergencia & 65 & $(80,3)$ & 178 & $(54,8)$ & $<0,001$ \\
\hline Hospitalización & 64 & $(79,0)$ & 234 & $(72,0)$ & 0,201 \\
\hline Gerencia* & 3 & $(3,7)$ & 15 & $(4,6)$ & 0,999 \\
\hline Sala de operaciones & 36 & $(44,4)$ & 94 & $(28,2)$ & 0,007 \\
\hline $\mathrm{UCl}^{*}$ & 3 & $(3,7)$ & 13 & $(4,0)$ & 0,999 \\
\hline Imágenes* & 1 & $(1,2)$ & 5 & $(1,5)$ & 0,999 \\
\hline Laboratorio y Patología* & 0 & $(0,0)$ & 9 & $(2,8)$ & 0,215 \\
\hline
\end{tabular}

* Prueba exacta de Fisher

UCl: Unidad de Cuidados Intensivos, MINSA: Ministerio de Salud del Perú, EsSalud: Seguro Social de Salud.

poner en práctica sus habilidades para entrevistar, negociar, solucionar problemas y comunicarse no solo con el paciente sino también con el familiar, haciéndolo parte activa del manejo del paciente ${ }^{(27)}$.

En esta investigación se encontró que los médicos que terminaron sus estudios de pregrado en ciudades fuera de LM tienen más probabilidad de ser víctimas de VLETA. Una hipótesis sobre el porqué de este hallazgo concierne a la diferencia de modelos de enseñanza, el contexto social de la enfermedad y al entrenamiento en la comunicación con el paciente ${ }^{(28)}$. Se propone también la diferencia sociocultural entre LM y otras ciudades peruanas, como un factor que generaría diferencias en los patrones de comunicación entre médico, paciente y familiares. Asimismo, las experiencias que viven los estudiantes de Medicina en LM, quienes estarían expuestos como testigos o víctimas a una agresión durante sus años de formación, los haría desarrollar estrategias para evitar ser agredidos.

Una variable no modificable asociada a VLETA es que la víctima sea varón. Al respecto, existen estudios con resultados contradictorios que dependerían del contexto cultural y religioso en donde se desarrollaron. Nuestro resultado se contrapone con lo reportado por 
Tabla 4. Factores asociados con violencia psicológica tipo amenaza durante los últimos doce meses en médicos de hospitales de Lima Metropolitana, $2014(n=406)^{*}$

\begin{tabular}{|c|c|c|c|c|}
\hline \multirow{2}{*}{ Características } & \multicolumn{2}{|c|}{ Modelo crudo* } & \multicolumn{2}{|c|}{ Modelo ajustado* $\dagger$} \\
\hline & RP & (IC 95\%) & $\mathbf{R P a}$ & (IC 95\%) \\
\hline Varón & 1,81 & $(1,28-2,96)$ & 1,66 & $(1,05-2,76)$ \\
\hline Egresó de universidad de provincia & 1,85 & $(1,15-2,54)$ & 1,54 & $(1,09-2,36)$ \\
\hline \multicolumn{5}{|l|}{ Lugar de trabajo } \\
\hline Clínica privada & 1,00 & Referencia & 1,00 & Referencia \\
\hline Hospital MINSA & 13,95 & $(3,42-85,95)$ & 7,98 & $(2,24-50,73)$ \\
\hline Hospital ESSALUD & 15,07 & $(3,85-93,18)$ & 8,68 & $(2,26-56,17)$ \\
\hline \multicolumn{5}{|l|}{ Área de trabajo } \\
\hline Emergencia & 2,73 & $(1,66-4,99)$ & 1,93 & $(1,24-3,55)$ \\
\hline Sala de operaciones & 1,70 & $(1,12-2,86)$ & 1,60 & $(1,07-2,34)$ \\
\hline Residente ${ }^{* *}$ & 1,80 & $(0,90-2,63)$ & - & - \\
\hline
\end{tabular}

* Estimación de intervalos de confianza mediante bootstrap no paramétrico con sesgo-corregido y acelerado con 1000 replicaciones para modelos lineales generalizado de familia Poisson link log con errores estándar robustos.

$† \quad$ El modelo incluyó todas las variables que mostraban asociación en sus respectivos modelos crudos.

** No fue incluido en el modelo ajustado, al no encontrase asociación en el modelo crudo.

RP: razón de prevalencias, RPa: razón de prevalencias ajustado, IC: intervalo de confianza, MINSA: Ministerio de Salud del Perú, EsSalud:

Seguro Social de Salud.

algunos estudios en los cuales el sexo de la víctima no generaría diferencias ${ }^{(13)} \mathrm{o}$ en otros donde la mujer tiene más probabilidad de sufrir violencia verbal $(13,14)$. Particularmente en Perú, el paciente o los familiares podrían conceder una mayor responsabilidad y expectativas sobre el médico varón, por lo que de no obtener lo esperado, podría incurrirse en actos violentos. Además, podrían existir diferencias por sexo en las áreas quirúrgicas y emergencia, donde es más frecuente el desempeño de médicos varones.

Nuestro estudio tiene limitaciones. Primero, la selección de la muestra fue no probabilística, lo cual podría conllevar a presentar un sesgo de selección, pues aceptar participar pudo corresponder a tener mayor interés en el tema por haber sido víctima de VLETA, con lo que se generaría sobreestimación de su frecuencia. Sin embargo, durante la ejecución del estudio se informó a los médicos sobre la importancia de participar, incluso sin haber sido víctima de VLETA y se invitó a la mayor cantidad de ellos. Adicionalmente, dado que el muestreo fue por conveniencia, esto llevó al no cumplimiento de supuestos para distribuciones poblacionales y, además, solo se reportó un caso de VLETA en el subsector privado; por lo que se utilizó bootstrap, por ser una técnica que permite obtener mejores estimaciones en este tipo de casos ${ }^{(18)}$; asimismo, ya que se desarrolló un modelo de asociación, el efecto negativo del muestreo no probabilístico es reducido ${ }^{(30)}$. Igualmente, si bien existe la posibilidad de un sesgo de selección del participante del estudio, pues un médico que labora en dos lugares hubiese podido ser seleccionado en otra IPRESS de otro subsector, el hecho violento en los últimos doces meses -como variable de respuesta principal- está registrado para un lugar específico.
Segundo, se puede tener un sesgo de información debido que se trata de un reporte personal por lo que la información brindada se afectaría por el sesgo de memoria; además, no se exploró la frecuencia de veces que el profesional pudo ser víctima de VLETA en un determinado periodo. No obstante, se definieron intervalos de tiempo para medir la frecuencia: durante la práctica profesional, los últimos doce meses y el último mes. Asimismo, ciertas variables relacionadas con el agresor (nivel educativo, nivel social, nivel económico, grado de agresividad basal, entre otros) no fueron medidas, ya que solo se exploró la perspectiva de la víctima. Tampoco se midió la capacidad del médico para establecer adecuadas relaciones con su paciente, su nivel agresividad, rasgos de personalidad, umbral de tolerancia, entre otros factores podrían influir en la perpetración del hecho violento.

A pesar de lo expuesto, debe considerarse que el estudio es novedoso, puesto que es una de las primeras publicaciones desarrolladas en Latinoamérica que evalúa factores asociados. Se debe remarcar que esta investigación es uno de los primeros estudios que exploran este problema en servicios de salud hospitalarios en el sistema de salud peruano, con la inclusión tanto del subsector público como privado. En ese contexto, la VLETA más que constituir un problema podría ser un síntoma que representa el status quo de un sistema de salud fraccionado, con marcadas deficiencias e inequidades como el peruano ${ }^{(10,15)}$.

Asimismo, estos resultados son relevantes, puesto que brindan un marco para la formulación de políticas de detección y prevención de VLETAen el Perú, más cuando se han identificado grupos de mayor vulnerabilidad. 
Algunos factores asociados pueden ser susceptibles a cambios o a la implementación de estrategias basadas en la mejor evidencia disponible, incluyendo información proveniente de estudios primarios de intervención, revisiones sistemáticas y evaluaciones económicas ${ }^{(26)}$. Se sugiere, por ejemplo, que las estrategias deben ser preventivas y de soporte. Las preventivas enfocadas a considerar algoritmos estandarizados de clasificación de pacientes, sobre todo en la emergencia, señalizaciones claras en los establecimientos de salud, información y concientización al paciente y sus familiares, mediadores en salas de esperas y unidades de seguridad con personal y recursos adecuados ${ }^{(27)}$. Paralelamente, las estrategias de soporte también deberían apoyar a la víctima tanto en la rehabilitación emocional - debido al alto impacto que pueden producir ${ }^{(28)}$ - como en relación a las leyes que regulen lo actos de violencia. Para ello, es pertinente que los establecimientos faciliten el reporte de estos hechos por parte del médico y se tenga un abordaje integral de este tipo de casos. Por otro lado, podría ser necesario que se realicen modificaciones legales que propugnen un incremento en la severidad de las penas en quienes ejerzan violencia sobre los profesionales de la salud, tal como ya se ha realizado en España ${ }^{(30)}$
Finalmente, estos hallazgos constituyen una base para el desarrollo de futuras investigaciones, las cuales incorporen la medición de variables del médico y del agresor, que cuenten con muestreo representativo nacional, así como la puesta en marcha de análisis longitudinales y estudios de intervención.

\section{Fuentes de Financiamiento: autofinanciado}

Conflictos de interés: los autores declaran no tener conflictos de interés. Uno de los autores labora al momento de la publicación en la Superintendencia Nacional de Salud (SUSALUD), no obstante, el presente manuscrito no constituye una posición oficial de SUSALUD.

Contribuciones de autoría: XTF, EMH y PMT participaron de la concepción, diseño del estudio, interpretación de resultados, XTF, EM, RA, MM, MS y JT participaron de la recolección de datos, XTF redactó el borrador del manuscrito, EMH y PMT realizaron el análisis de datos, todos los autores hicieron la revisión crítica del manuscrito, aprobaron la versión a publicar y se responsabilizan del contenido del artículo.

Agradecimientos: a Bruce Peña Martínez, Marcia Oliveira Villanueva, Giuliana Matos Ibérico y Ricardo Carpio Guzmán por su apoyo en la recolección de datos.

\section{REFERENCIAS BIBLIOGRÁFICAS}

1. Organización Internacional del Trabajo, Consejo Internacional de Enfermeras, Organización Mundial de la Salud, Internacional de Servicios Públicos. Directrices marco para afrontar la violencia laboral en el sector de la salud. Ginebra: Organización Internacional del Trabajo; 2002

2. Organización Internacionaldel Trabajo. Repertorio de recomendaciones prácticas sobre la violencia en el lugar de trabajo en el sector de los servicios y medidas para combatirla. Ginebra: Oficina Internacional del Trabajo; 2003.

3. Alexander C, Fraser J. Occupational violence in Australian healthcare setting: implications for managers. J Healthc Manag. 2004;49(6):377-90.

4. Buvinic M, Morrison A, Shifter M. La violencia en América Latina y el Caribe: un marco de referencia para la acción. Washington D.C.: Banco Interamericano de Desarrollo; 1999.

5. Di Martino V. Relationship between work stress and workplace violence in the health sector. Ginebra: Organización Internacional del Trabajo; 2002.

6. Cooper CL, Swanson N. Workplace violence in the health sector: state of the art. Geneva: OIT, OMS, CIE, ISP; 2002.

7. Hahn S, Zeller A, Needham I, Kok G, Dassen T, Halfens R. Patient and visitor violence in general hospitals: a systematic review of the literature. Aggress Violent Behav. 2008; 13(6):431-41. doi: 10.1016/j. avb.2008.07.001

8. Braga F, Prats M, Luna D, Melamud A, Flitchtentrei D. Agresiones contra Médicos [Internet]. Buenos Aires: IntraMed; 2011 [citado el 19 de setiembre 2011] Disponible en: http://www.intramed.net/UserFiles/ pdf/36397.pdf

9. Tuya-Figueroa X, Mezones-Holguín E. Violencia contra médicos: un problema por considerar en la investigación de recursos humanos en salud. Rev Peru Med Exp Salud Publica. 2012;29(1):149-67.

10. Muñoz A, Valdivia-Fernandez B, Velasquez-Lazo C, Valdivia-Rodriguez M, Arroyo-Tovar GY, Toia-Larsen $\mathrm{M}$, et al. Violencia contra médicos en hospitales de Arequipa, Perú 2016. Acta Med Peru. 2016;33(2):99-104.

11. Ancalli-Calizaya F, Cohaila G, Maquera-Afaray J. Agresiones contra trabajadores de salud en Tacna, Perú.
Rev Peru Med Exp Salud Publica. 2012;29(3):415-6.

12. Arimatsu M, Wada K, Yoshikawa T, Oda S, Taniguchi H, Aizawa Y, et al. An epidemiological study of work-related violence experienced by physicians who graduated from a medical school in Japan. J Occup Health 2008;50(4):357-61.

13. Ness GJ, House A, Ness AR. Aggression and violent behavior in general practice population bases survey in the north of England. BMJ. 2000;320(7247):14478.

14. Koritsas S, Coles J, Boyle M, Stanley J. Prevalence and predictors of occupational violence and aggression towards GPs: a cross-sectional study. Br J Gen Pract. 2007;57(545):967-70. doi: 10.3399/096016407782604848

15. Sánchez-Moreno F. El sistema nacional de salud en el Perú. Rev Peru Med Exp Salud Publica. 2014;31(4):747-53.

16. Caballero P, Yagui $M$, Espinoza $M$, Castilla T, Granados A, Velásquez $\mathrm{A}$, et al. Prioridades regionales y nacionales de investigación en salud, Perú 2010-2014: un proceso con enfoque participativo y descentralista. Rev Peru Med Exp Salud Publica. 2010;27(3):398-411. 
17. International Labour Organization, World Health Organization, International Council of Nurse, Public Services International. Workplace violence in the health sector country case studies research instruments survey questionnaire. Geneva: ILO, WHO, ICN, PSI; 2003.

18. Davison AC, Hinkley DV. Bootstrap methods and their application. 1a ed. Cambridge: Cambridge University Press; 1997.

19. Vela C. Evaluación de la calidad del servicio de emergencia de adultos del HNERM-EsSalud, desde el punto de vista de los usuarios, año 2005 [tesis doctoral]. Lima: Universidad Nacional Mayor de San Marcos; 2010.

20. Belayachi J, Berrechid K, Amlaiky F, Zekraoui A, Abouqal R. Violence toward physicians in emergency departments of Morocco: prevalence, predictive factors, and psychological impact. J Occup Med Toxicol. 2010;5:27. doi: 10.1186/1745-66735-27

21. Mirza NM, Amjad AI, Bhatti AB, tuz Zahra Mirza F, Shaikh KS, Kiani J, et al. Violence and abuse faced by junior physicians in the emergency department from patients and their caretakers: a nationwide study from Pakistan. J Emerg Med. 2012;42(6):727-33. doi: 10.1016/j. jemermed.2011.01.029

22. Hobbs FD, Keane UM. Agression against doctors: a review. J R Soc Med. 1996;89(2):69-72.

23. Landau SF, Bendalak Y. Personnel exposure to violence in hospital emergency wards: a routine activity approach. Aggress Behav. 2008;34(1):88-103.

24. Mejia CR, Allpas-Gomez HL, Caceres OJ, Red GIS Peru. Factores socio-laborales asociados al maltrato psicológico, físico y sexual en el personal de salud en dos hospitales de Lima, Perú. Arch Med. 2016;12(1):e6.

25. Violence against doctors: Why China? Why now? What next? Lancet 2014; 383(9922):1013. doi: 10.1016/S01406736(14)60501-8

26. Touzet S, Cornut PL, Fassier JB, Le Pogan MA, Burillon C, Duclos A. Impact of a program to prevent incivility towards and assault of healthcare staff in an ophthalmological emergency unit: study protocol for the PREVURGO On/Off trial. BMC Health Serv Res. 2014;14:221. doi: $10.1186 / 1472-6963-14-221$
27. Saeki K, Okamoto N, Tomioka K, Obayashi K, Nishioka H, Ohara K, et al. Work-related aggression and violence committed by patients and its psychological influence on doctors. J Occup Health. 2011;53(5):356-64

28. Shi J, Wang S, Zhou P, Shi L, Zhang Yu, Bai F, et al. The frequency of patientinitiated violence and its psychological impact on physicians in China: a cross-sectional study. PLoS One. 2015;10(6):e0128394.

29. Rothman KJ, Gallacher JE, Hatch EE. Why representativeness should be avoided. Int $\mathrm{J}$ Epidemiol. 2013;42(4):1012-4.

30. Marinas-Sanz R, Begoña MartínezJarreta B, Casalod Y, Bolea M. Las agresiones a profesionales sanitarios en España: análisis tras la reciente modificación del Código Penal. Med Clin (Barc). 2016;147(1):35-52.

\footnotetext{
Correspondencia:Edward Mezones-Holguin Correo electrónico:emezones@gmail.com Dirección: Prolongación Primavera 2390, Monterrico, Santiago de Surco. Lima, Perú. Teléfono: (+511) 3118888
}

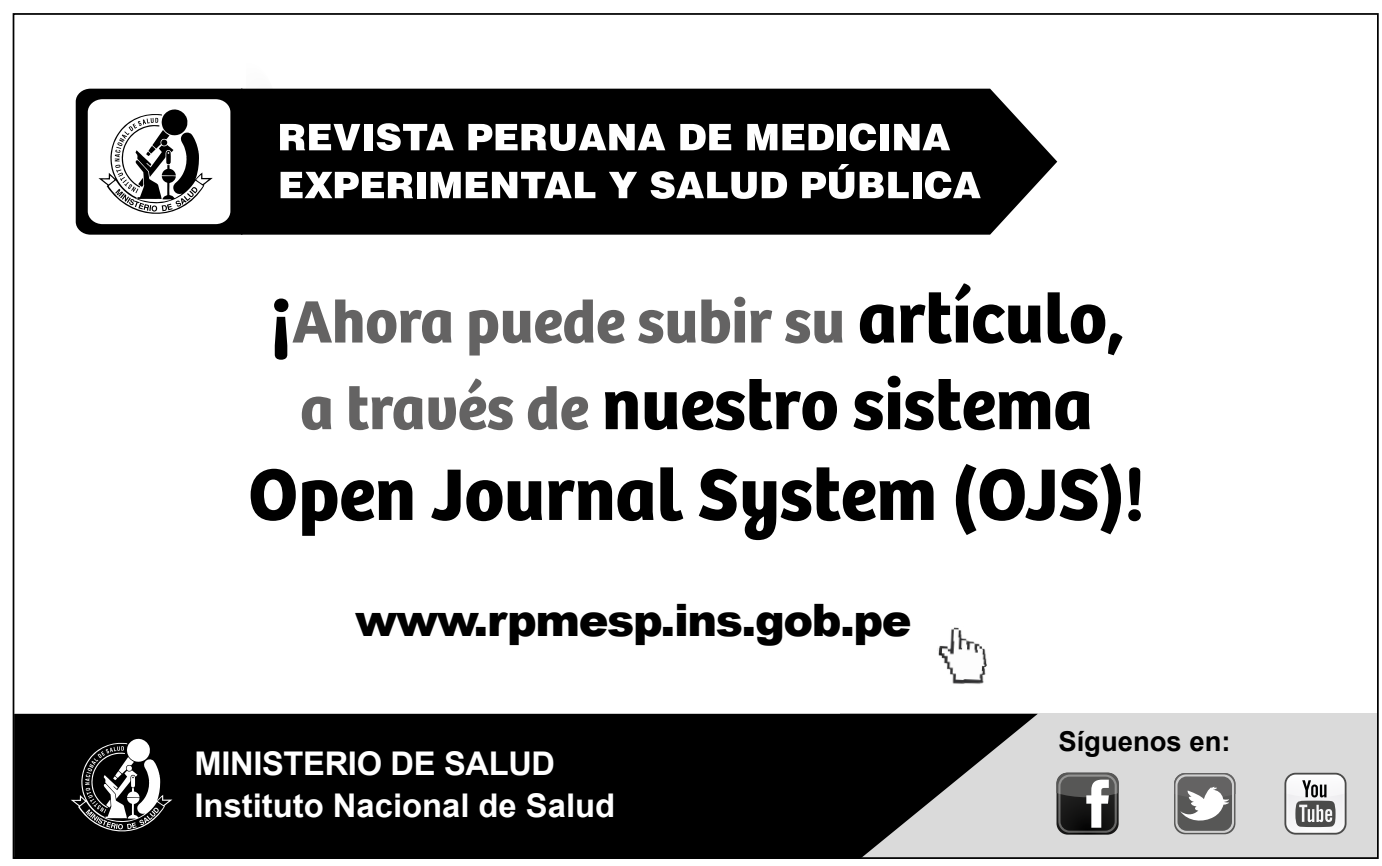

